\title{
Using Fuzzy Neural Networks to Improve Prediction of Expert Systems for Detection of Breast Cancer
}

\author{
Augusto Júnio Guimarães ${ }^{1}$, Vinicius Jonathan Araújo ${ }^{1}$, Lucas de Oliveira Batista ${ }^{1}$, \\ Paulo Vitor Campos Souza ${ }^{1,2}$, Vanessa Araújo ${ }^{1}$, Thiago Silva Rezende ${ }^{1}$ \\ ${ }^{1}$ Centro Universitário UNA - Betim \\ Av. Gov. Valadares, 640 - Centro, Betim - MG, 32510-010 \\ ${ }^{2}$ Centro Federal de Educação Tecnológica de Minas Gerais (CEFET-MG) \\ Av. Amazonas, 5.253, Nova Suiça, Belo Horizonte, MG, Brasil. CEP: 30.421-169 \\ augustojunioguimaraes@gmail.com/vinicius.j.s.a22@hotmail.com/
lobatista@outlook.com.br/goldenpaul@informatica.esp.ufmg.br/ \\ v.souzaaraujo@yahoo.com.br/sillvarezendethiago@hotmail.com
}

\begin{abstract}
Breast cancer is one of the most affected diseases worldwide, with 1.38 million new cases and 458 thousand deaths from the disease each year, according to the World Health Organization. In this article, we use a hybrid artificial intelligence model based on concepts of neural networks and fuzzy systems to improve the predictive results of expert systems capable of working with numerical datasets allowing in addition, an assertive prediction in addition to an assertive prediction, to increase the interpretability of the results through fuzzy neural network architecture, capable of creating nebulous rules that allow greater understanding of these the template. The results of the tests performed when compared to other models commonly used for this purpose corroborate that the presented model maintains the amplification of the accuracy and is able to create nebulous rules that allow a greater interpretability to the problem.
\end{abstract}

Resumo. O câncer de mama é uma das patologias que mais acomete as mulheres em todo o mundo, sendo 1,38 milhões de novos casos e 458 mil mortes pela doença por ano de acordo com a OMS. Existem várias áreas de estudo focadas na detecção e tratamento desse tipo de câncer, pois a detecção precoce contribui para o tratamento adequado e eficaz. Neste artigo é utilizado de um modelo híbrido de inteligência artificial baseado em conceitos de redes neurais e sistemas nebulosos para melhorar os resultados preditivos de sistemas especialistas capazes de trabalharam com bases de dados numéricas permitindo além de uma previsão assertiva, aumentar a interpretabilidade dos resultados através da arquitetura da rede neural nebulosa, capaz de criar regras nebulosas que permitem maior compreensão sobre a utilização do modelo. Os resultados dos testes realizados quando comparados a outros modelos comumente utilizados para esse fim corroboram que o modelo apresentado mantém a amplificação da precisão e é capaz de criar regras nebulosas que permitem uma maior interpretabilidade ao problema. 


\section{Introdução}

A composição do corpo humano é caracterizada por diversos tipos de tecidos no qual são compostos por uma diversidade de células. O câncer é definido pelo crescimento acelerado e desordenado dessas células, causando um tumor, independente de qual parte do corpo se encontra. Quando não realizado o diagnóstico precoce e/ou não realizado um tratamento, esse tumor tende a crescer agressivamente, tornando-se maligno. É caracterizado como câncer de mama, quando esse processo ocorre na região da mama, geralmente desenvolve-se nas células do revestimento dos canais mamários e dos lóbulos onde é produzido o leite [Bergamasco and Angelo 2001]. Atualmente encontramos de formas variadas, técnicas e procedimentos para a detecção do câncer. O auto teste de mama, é o primeiro exame que a mulher pode realizar em seu corpo, nele a mulher realiza a apalpação da sua mama buscando detectar o aparecimento de massas incomuns. Outro procedimento bastante utilizado é a mamografia de diagnóstico, que utiliza raios-X para produzir várias imagens (mamogramas), logo permitem detectar tumores e outras irregularidades na mama que não são identificados apenas por apalpação [Seabra and Lourenço 2013] . Temos também a biópsia que consiste em recolher uma pequena quantidade de tecido ou de células da área suspeita, que depois é analisada ao microscópio por um patologista [Ribeiro-Silva 2012]. Por fim, outro grande método empregado é a análise de sangue, permitindo a realização de vários exames para a verificação das células do corpo, avaliando se elas estão com valores esperados. Para auxiliar nesse tipo de tratamento que pode ser altamente invasivo e prejudicial as mulheres, diversas pesquisas vêm sendo realizadas com o intuito de coletar bases de dados para auxiliar na predição de câncer de mama. O trabalho proposto por [Patrício et al. 2018], apresenta características relevantes de um grupo de mulheres que são avaliadas acerca da presença de células cancerígenas em suas mamas. Para aspectos de predição, o autor utilizou alguns fatores coletados das mulheres. Com o objetivo de definir as melhores características utilizadas, aplicou técnicas de seleção de características e relevância estatística de atributos. Finalizando, para comprovar a capacidade preditiva, foram executados testes de classificação de padrões utilizando modelos de máquina de vetores de suporte (SVM), modelos de regressão linear (LR) e de árvores de decisões.

Esse trabalho tem como proposta a utilização de uma estrutura híbrida que une conceitos de redes neurais e sistemas nebulosos. Com base em sistemas de regras nebulosas, desenvolvido para melhorar interpretabilidade do sistema de predição de câncer de mama, baseado em estudo proposto por [Patrício et al. 2018] onde o modelo é capaz de gerar interpretabilidade nos resultados obtidos pela rede. Propõe-se uma nova forma de partição dos dados de entrada, a utilização de neurônios lógicos nebulosos andneuron e unineuron, auxiliando na definição da topologia da rede utilizaremos um algoritmo baseado na teoria da regularização para encontrarmos os neurônios mais significativos ao modelo. A utilização de redes neurais nebulosas vem sendo empregada em diversos ramos, como economia [Bakirtzis et al. 1995], na área de reconhecimento de fonemas [Kasabov et al. 1998], predição de características voltadas a arritmia cardíaca [Özbay et al. 2006], seleção de características [Silva et al. 2013] e previsão de vazão de chuvas [Ballini et al. 2003]. O treinamento da rede neural nebulosa [Souza 2018] proposta nesse artigo busca realizar testes de classificações binárias, com a base de dados disponibilizada em [Patrício et al. 2018] para criar as regras de conhecimento do sistema de predição do câncer de mama. A utilização da máquina de aprendizado ex- 
tremo [Huang et al. 2006] facilita a criação, atualização dos pesos da camada de saída e parâmetros do sistema, contribuindo para que a previsão do sistema inteligente ocorra de forma mais precisa, rápida e adaptativa. O artigo se encontra organizado na seguinte sequência: Na seção em sequência temos o referencial teórico, com uma revisão de conceitos importantes envolvida no desenvolvimento do trabalho. Na seção 3 são apresentadas a descrição do processo de utilização do modelo híbrido de inteligência artificial com o objetivo de melhorar os resultados preditivos do sistema especialista para a predição do câncer de mama, com detalhamentos de processos e conceitos específicos utilizados. A seção 4 expõe o funcionamento dos testes realizados, bem como os experimentos utilizados para as avaliações e seus respectivos resultados. Finalmente na seção 5 são apresentadas as conclusões finais.

\section{Referencial Teórico}

\subsection{Conceitos Sobre o Câncer de Mama}

O Câncer de mama é o desenvolvimento de um tumor maligno na região mamária. O principal sinal de sua presença é o aparecimento de um nódulo na região dos seios, alterações na forma ou aparência da mama, ou até uma sensibilidade e secreção de um líquido pelo mamilo. Excluindo o câncer de pele, o tumor maligno na mama é o mais comum diagnosticado entre as mulheres nos Estados Unidos, sendo responsável por quase um em cada três tipos dessa doença. É também a segunda principal causa de morte entre mulheres após o câncer de pulmão [DeSantis et al. 2014].

Ao que tudo indica, o câncer de mama é o resultado da interação de fatores genéticos com estilo de vida, hábitos reprodutivos e meio ambiente. Entre os fatores de risco para o seu desenvolvimento estão o sexo feminino, a idade, obesidade, falta de exercício físico, o consumo de álcool, a terapia de reposição hormonal durante a menopausa, radiação ionizante, idade precoce da primeira menstruação, ter filhos em idade tardia ou não ter tido filhos [Rossi and dos Santos 2003]. Todos os cânceres de mama têm origem genética. Acredita-se que 90\%-95\% deles sejam esporádicos (não-familiares) e decorram de mutações somáticas que se verificam durante a vida, e que 5\%-10\% sejam hereditários (familiares) devido à herança de uma mutação germinativa ao nascimento [Bergamasco and Angelo 2001]. A detecção do câncer de mama é uma estratégia importante para possibilitar um tratamento antecipado e proporcionar uma probabilidade maior de um bom resultado no combate à doença.

Em pessoas a quem foi diagnosticado o câncer, estão disponíveis uma série de tratamentos, incluindo cirurgia, radioterapia, terapia hormonal e terapia direcionada [Mineo et al. 2013]. O prognóstico para o câncer da mama varia em função do seu tipo, extensão da doença e idade da pessoa. A taxa de sobrevivência em países desenvolvidos é elevada, sendo mais reduzida nos países em vias de desenvolvimento. Quando se considera todo o mundo, o câncer da mama é a causa mais comum em mulheres, correspondendo a 25\% de todos os casos [DeSantis et al. 2014]. O câncer de mama é o tipo de câncer que mais afeta as mulheres, contudo, a doença também pode ocorrer em homens [Freitas et al. 2008], porém de forma mais rara, pois de acordo com as estatísticas, para cada 1 homem diagnosticado com câncer de mama, 100 mulheres apresentam a patologia. A figura 1 apresenta características do câncer em uma mama. 


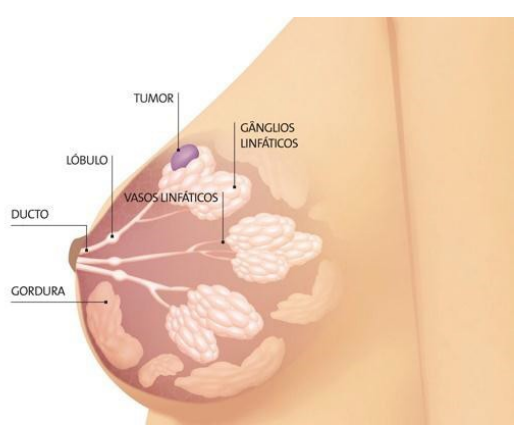

Figura 1. Câncer de mama. Disponível em: http://rochalima.com.br/blog/cancermama-tratamentos/

\subsection{Redes Neurais Artificiais}

As redes neurais artificiais são modelos que utilizam em suas estruturas o neurônio lógico, buscando simular o processamento de informação do cérebro humano através de uma rede de diversos neurônios artificiais interligados, que se unem por meio de conexões sinápticas. De uma forma simplificada, uma rede neural artificial pode ser vista como um grafo onde os nós são os neurônios e as ligações fazem a função das sinapses. As redes neurais artificiais se diferenciam pela sua arquitetura e pela forma como os pesos associados às conexões são ajustados durante o processo de aprendizado. O aprendizado é a forma com que a rede neural capta as informações fornecidas pelas entradas, através das conexões e dos pesos sinápticos tomam decisões acerca do tema central da base de dados. A arquitetura de uma rede neural restringe o tipo de problema no qual a rede poderá ser utilizada, e é definida pelo número de camadas (camada única ou múltiplas camadas), pelo número de nós em cada camada, pelo tipo de conexão entre os nós (feedforward ou feedback) e por sua quantidade de respostas [Haykin and Network 2004]. A figura 2 apresenta a estrutura de uma rede neural com múltiplas camadas. Ela também evidencia os neurônios e suas ligações sinápticas.

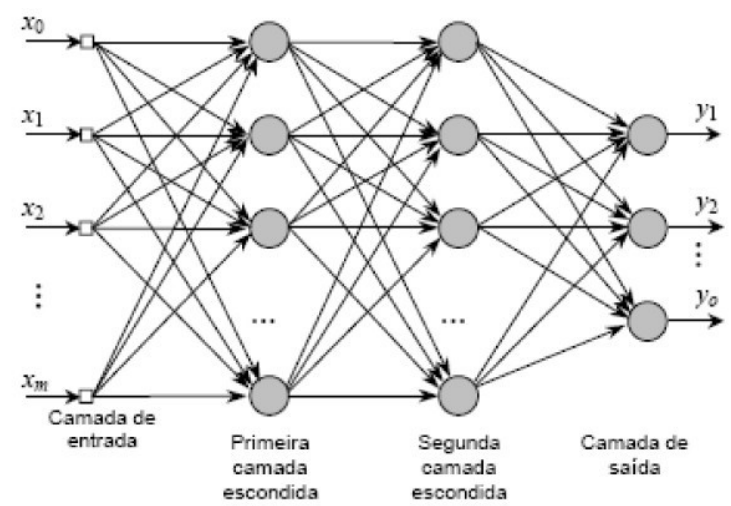

Figura 2. Rede neural artificial de múltiplas camadas e saídas. Disponível em: http : //www.scielo.br/scielo.php?script $=$ sciarttextpid $=$ S0104 -77602013000200012

\subsection{Sistemas Nebulosos}

O uso dos sistemas nebuloso faz-se necessários em casos onde a abordagem clássica torna-se inviável para a resolução de um problema devido à natureza de sua complexidade [Calvo 2007]. Os métodos mais conhecidos são passíveis de alterações bruscas 
para a resolução de problemas devido à simplificação do modelo real, porém os sistemas nebulosos possuem recursos (funções de pertinência, regras e operadores de agregação) que possibilitam a aproximação mais fiel ao modelo real, evitando que a solução gerada pelo sistema nebuloso destoe consideravelmente da realidade esperada. A figura 3 apresenta os principais elementos que compõem a lógica fuzzy: suas entradas, o processo da transformação de entradas em elementos fuzzy, a criação de conjuntos fuzzy de entrada, o conjunto de regras e inferências, a obtenção dos conjuntos fuzzy de resposta, a defuzzyficação que é tornar os valores obtidos de acordo com as entradas do sistema e as saídas de forma esperada.

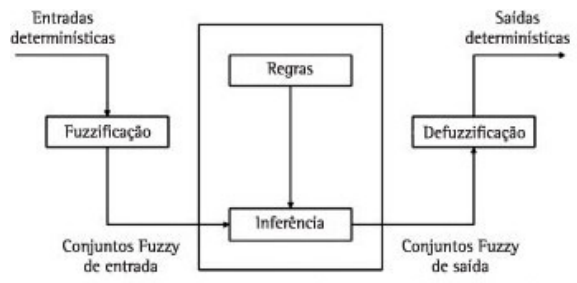

Figura 3. Conceitos presentes na lógica nebulosa (fuzzy). Imagem disponívl em: http://www.scielo.br/scielo.php6

\subsection{Neurônios Lógicos Nebulosos}

Dentre os vários estudos realizados para simular o comportamento do neurônio humano, destacamos os que buscaram agregar a natureza nebulosa ao modelo de neurônio artificial, acrescentando-lhe a habilidade de tratar informações imprecisas. A esse neurônio chamamos de neurônio nebuloso [Jang et al. 1997]. Os neurônios nebulosos do tipo III (descritos por equações lógicas nebulosas) possuem algumas variações de modelos que combinam os neurônios nebulosos do tipo II (com entradas nebulosas combinados com pesos nebulosos) e III. A esses neurônios chamamos de neurônios lógicos. Foram propostos por [Savic and Pedrycz 1991] e apresentam uma estrutura similar aos neurônios do tipo III, excetuando o fato de que os pesos de conexão $w_{i}$ serem associados a cada uma das entradas $x_{i}$. Assim o neurônio lógico executa um mapeamento no espaço formado pelo produto cartesiano entre o espaço de entrada e o espaço dos pesos no intervalo unitário, isto é X X W - [0,1] [Jang et al. 1997].

Podemos citar como neurônios lógicos nebulosos and e or, além de um baseado em uninorma, que é uma generalização de t-norma e s-norma [Pedrycz 1991], chamando unineuron. Quando empregamos o neurônio lógico and utilizamos uma s-norma como operador de ponderação, enquanto o operador de agregação é implementado por uma $t$ norma, diferentemente de quando utilizamos o neurônio lógico or, que é realizado através da utilização de uma t-norma como operador de ponderação e uma $s$ - norma como operador de agregação [Jang et al. 1997]. Já o unineuron foi tratado inicialmente como a extensão dos neurônios lógicos and e or [Savic and Pedrycz 1991], onde podemos destacar que o processamento do neurônio acontece em dois níveis. No primeiro nível os sinais de entrada são individualmente combinados com os pesos, já no segundo nível uma ação global é realizada sobre os resultados de todas as combinações do primeiro nível [Lemos et al. 2010]. Considere como sinal de entrada $\mathbf{a}\left[a_{1}, a_{2}, \ldots a_{n}\right]$ e os pesos $\mathbf{w}$ $\left[w_{1}, w_{2}, \ldots w_{n}\right]$ para $a_{i} \in[0,1]$ e $w_{i} \in[0,1]$ para $i$ de $1, \ldots, n$. A agregação realizada pelos 
neurônios lógicos nebulosos and e or, onde os sinais de entrada são combinados individualmente com os pesos e realizada a agregação global posterior podem ser definidas como se segue [Savic and Pedrycz 1991]:

$$
\begin{gathered}
z=O R(a, w)=S_{i=1}^{n}\left(a_{i} t w_{i}\right) \\
z=A N D(a, w)=T_{i=1}^{n}\left(a_{i} s w_{i}\right)
\end{gathered}
$$

onde $\mathrm{T}$ e $\mathrm{t}$ são a representação de uma t-norma e $\mathrm{S}$ e s uma s-norma. Já para o unineuron [Lemos et al. 2010] descreveram os passos para realizar as funções do neurônio:

- Transformar cada par $\left(a_{i}, w_{i}\right)$ em um único valor $b_{i}=h\left(a_{i}, w_{i}\right)$;

- Calcular a agregação uninorma dos valores transformados $\mathbf{U}\left(b_{1}, b_{2}, \ldots, b_{n}\right)$, onde $n$ é o número de entradas.

A função $h$ é responsável por transformar as entradas e os pesos correspondentes em valores transformados individuais [Lemos et al. 2010]. Uma formulação para a função h pode ser visualizada:

$$
h(w, a)=w * a+w * g
$$

Utilizando a agregação ponderada relatada em [Seabra and Lourenço 2013] podemos escrever o unineuron [Lemos et al. 2010]:

$$
z=U N I(w, x ; g)=U_{i=1}^{n} h(w, x)
$$

\section{REDE NEURAL NEBULOSA PARA DETECÇÃo DO CÂNCER DE MAMA}

\subsection{Arquitetura e treinamento da rede neural nebulosa para problemas de classificação binária.a}

A área inteligência computacional apresenta grandes avanços no desenvolvimento de técnicas, modelos que simulam o comportamento humano atuando em processos e sistemas, com destaque para redes neurais artificiais, sistemas nebulosos e seus modelos híbridos, com grande quantidade de novas aplicações sendo propostas na literatura. Um dos principais objetivos das pesquisas em inteligência computacional é criar e modelar sistemas computacionais que emulem características específicas dos seres humanos, como aprendizado, intuição, raciocínio lógico, classificação e regressão [Haykin and Network 2004]. Um dos modelos matemáticos utilizados para realizar simulações do comportamento humano são as redes neurais nebulosas, que são redes neurais compostas em sua estrutura de neurônios nebulosos. Esses modelos diferem dos modelos tradicionais de redes neurais e de sistemas nebulosos devido à cooperação entre os conceitos de redes neurais com as ideias oriundas da teoria dos conjuntos nebulosos, gerando um modelo capaz de utilizar a capacidade de aprendizado das redes neurais em conjunto a eficiência do trato a imprecisão e a interpretação que possuem os sistemas nebulosos [Savic and Pedrycz 1991].

A arquitetura da rede neural nebulosa utilizada nesse trabalho é a mesma relatada em [11]. Portanto a rede neural nebulosa a ser utilizada nesse artigo utiliza na primeira camada a divisão de espaço de entrada em formato de grid chamado FIS [Souza 2018] , isto é, faz o processo de fuzzificação relatado na figura 3 utilizando a divisão do espaço 
de entrada em um número $\mathrm{M}$ de funções de pertinência nebulosas do tipo Gaussianas e Triangulares igualmente esparsadas e centradas em 0.5. Esses tipos de divisões através de funções de pertinência com as mesmas características podem auxiliar na interpretação linguística. O Sistema de Inferência fuzzy (fuzzy Inference System - FIS) é uma estrutura computacional baseada nos conceitos de teoria de conjuntos nebulosos, regras fuzzy do estilo $S E$... ENTÃO e raciocínio fuzzy onde sua estrutura possui três camadas conceituais: uma base de regras, uma base de dados e uma base de raciocínio [Jang et al. 1997]. Em [Jang et al. 1997] explica que o FIS é capaz de realizar um mapeamento não linear a partir de seu espaço de entrada para o espaço de saída. Este mapeamento é acompanhado por um número de regras fuzzy SE... ENTÃO, onde cada uma delas descreve o comportamento local do mapeamento. Os antecedentes nas regras de um sistema de inferência nebuloso implementam uma partição nebulosa multidimensional, que pode ser em grid, árvore de decisão ou por agrupamento, no espaço das variáveis de entrada de um modelo. Nesse artigo foi utilizada uma avaliação da seguinte forma para filtrar os neurônios que sairão da primeira camada: Se o número de características do problema for menor do que seis e o número de funções de pertinência escolhidos são menores ou igual a três, utilizase a abordagem inicialmente proposta em [Jang et al. 1997] Essa escolha se dá devido a diversos testes realizados anteriormente que verificavam que a capacidade de criação de regras nebulosas tornava o problema muito mais complexo do que era necessário. Se a premissa não for atendida a quantidade de neurônios da primeira camada é definida utilizando uma proposta modificada de [Jang et al. 1997], onde existe um filtro limitador de criação aleatória de 500 neurônios nebulosos baseados no espaço de entrada. O número 500 foi arbitrado após vários testes de desempenho do algoritmo utilizando conceitos de validação cruzada com diversas combinações de números de características e funções de pertinência. A figura 4 a seguir explica como funciona a partição dos dados de entrada conforme a partição utilizada.

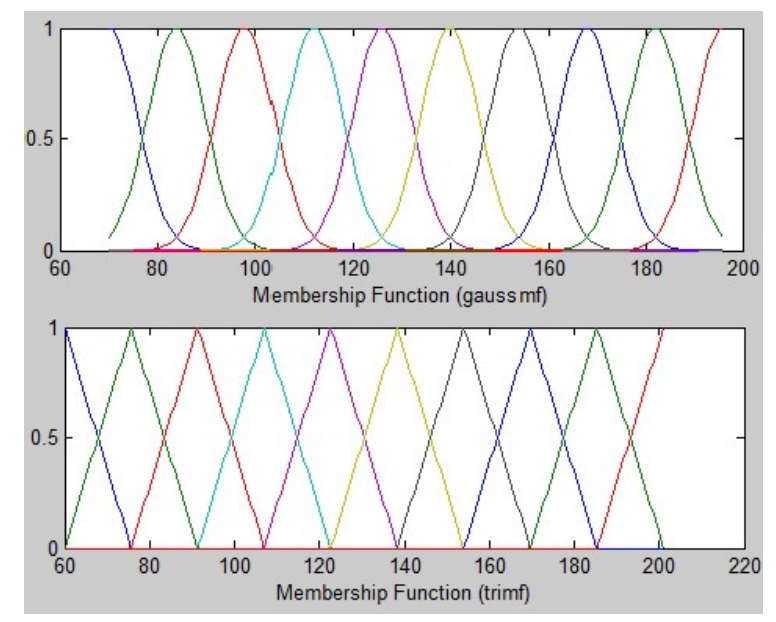

Figura 4. Divisão do espaço de entrada realizado por funções de pertinência gaussianas e triangulares

Considerando $v 1$ e $v 2$ como duas características presentes nos dados coletados dos pacientes do estudo, podemos inferir regras linguísticas do tipo: Se $v 1$ é pequeno e v2 é pequeno, portanto y é 1 , da mesma forma que se $v 1$ e $v 2$ foram grandes, a classe de y é 2. Dentro desse modelo de regras nebulosas, podem ser substituídos os valores de $v l$ e 
$v 2$ por elementos de uma base de pesquisa sobre câncer e as respostas 1 e 2 por pessoas com câncer e pessoas sem câncer. Nesse contexto pode-se aplicar por exemplo o valor de $v 1$ uma dimensão como idade e $v 2$ como o IMC das mulheres. Porém, conforme descrito em [Patrício et al. 2018], os agrupamentos das bases foram feitos com base em regras de relevância estatística e nos resultados finais dos testes com classificadores não foi calculada a acurácia de teste e a interpretabilidade dos resultados, dando destaque somente ao valor de AUC de sensibilidade de especificidade. Na segunda camada existem neurônios lógicos nebulosos que utilizam conceitos de unineuron proposto em [Lemos et al. 2010], que realizam a agregação dos pesos e das funções de ativação dos neurônios nebulosos da primeira camada. Nesse artigo resolveu-se avaliar as abordagens regularizada que utiliza o Bolasso [Bach 2008] e não regularizada do modelo de [Souza 2018], para verificar se a quantidade de neurônios afeta a acurácia do modelo. Os pesos da segunda camada são definidos utilizando os conceitos de máquina de aprendizado extremo [Huang et al. 2006] que atuam de maneira eficiente na geração rápida dos pesos, diferentemente de métodos que trabalham com retro-propagação para atualizar a arquitetura das redes. Em modelos que trabalham com grande massa de dados, essa abordagem torna- se viável devido a sua natureza mais simples de geração dos pesos utilizando conceitos da pseudo-inversa. O modelo utiliza um neurônio artificial simples, que pode ser visto como singleton. A arquitetura da rede neural nebulosa utilizada no artigo é apresentada na figura 5.

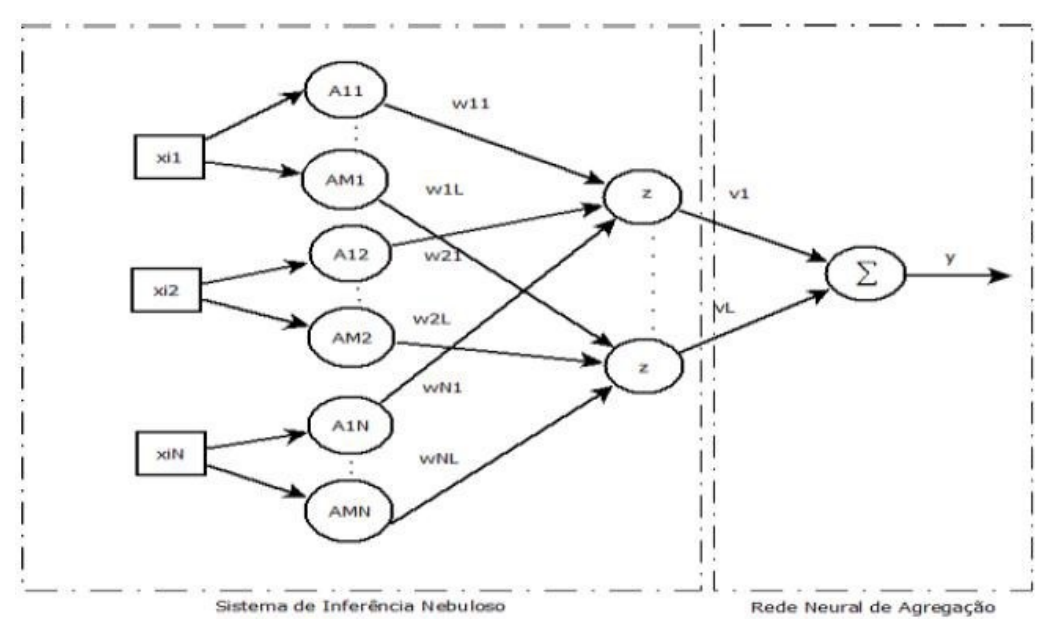

Figura 5. Estrutura da rede neural nebulosa utilizada no artigo [de Campos Souza and Guimarães ]

\section{BASE DE DADOS SOBRE PESQUISA DE PREDIÇÃO DE CÂNCER DE MAMA}

A pesquisa para a predição do câncer de mama apresentado em [Patrício et al. 2018] foi realizado após um estudo no qual foram utilizados 9 atributos como parâmetros, na participação de 166 pacientes. Esses parâmetros foram organizados e submetidos em 3 algoritmos de classificação: regressão logística, florestas aleatórias e máquinas de vetores de suporte. Os valores coletados no estudo foram organizados utilizando letras e números para representar características, onde $V 1=$ Glicose, $V 2=$ Resistina, $V 3=$ Idade, $V 4=$ IMC - índice de massa corporal, $V 5=$ HOMA - avaliação do modelo de homeostase para resistência à insulina, $V 6=$ Leptina, $V 7=$ Insulina, $V 8=$ Adiponectina e $V 9=\mathrm{MCP}-1$ - 
proteína quimiotática de monócitos-1. Para a modelagem, o autor dividiu as variáveis incluídas em 6 agrupamentos de acordo com técnicas de relevância estatística (V1-V2, V1V3, V1-V4, V1-V5, V1-V6,V1-V9). Cada combinação considerou como características preditores as dimensões mais significativas para o problema, calculada a partir de uma regra de seleção de características para poder determinar quais eram os fatores mais relevantes para determinar pacientes com probabilidade de câncer. Em correlação com a classificação, tem três algoritmos de avaliação, sendo eles: AUC, Sensibilidade e Especificidade. Onde a sensibilidade e especificidade consiste em respectivamente acertar quando pessoas doentes estão realmente doentes e não doentes quando não possuem a doença. Com base nos resultados, a presença ou não do câncer de mama em mulheres poderia ser prevista com sensibilidade variando entre $82 \%$ e $88 \%$ e especificidade variando entre $85 \%$ e $90 \%$. Indicando que a Resistina e a Glicose, em conjunto com a Idade e o IMC, podem ser consideradas um bom agrupamento para biomarcadores de câncer de mama, mesmo apenas estimando, como mostra os resultados na tabela 1.

\begin{tabular}{|c|c|c|c|c|}
\hline Variáveis & \multirow{2}{*}{ Avaliações } & \multicolumn{3}{|c|}{ Classificadores } \\
\cline { 2 - 5 } & & LR & RF & SVM \\
\hline \multirow{3}{*}{ V1V2 } & AUC & {$[0.76,0.81]$} & {$[0.70,0.75]$} & {$[0.76,0.81]$} \\
\cline { 2 - 5 } & Sensitividade & {$[0.75,0.81]$} & {$[0.75,0.82]$} & {$[0.81,0.86]$} \\
\cline { 2 - 5 } & Especificidade & {$[0.73,0.80]$} & {$[0.63,0.70]$} & {$[0.70,0.76]$} \\
\hline \multirow{3}{*}{ V1V3 } & AUC & {$[0.76,0.80]$} & {$[0.81,0.85]$} & {$[0.82,0.86]$} \\
\cline { 2 - 5 } & Sensitividade & {$[0.74,0.81]$} & {$[0.85,0.90]$} & {$[0.87,0.92]$} \\
\cline { 2 - 5 } & Especificidade & {$[0.74,0.80]$} & {$[0.72,0.78]$} & {$[0.78,0.83]$} \\
\hline \multirow{3}{*}{ V1V4 } & AUC & {$[0.79,0.83]$} & {$[0.84,0.88]$} & {$[0.87,0.91]$} \\
\cline { 2 - 5 } & Sensitividade & {$[0.72,0.78]$} & {$[0.80,0.86]$} & {$[0.82,0.88]$} \\
\cline { 2 - 5 } & Especificidade & {$[0.80,0.87]$} & {$[0.81,0.87]$} & {$[0.84,0.90]$} \\
\hline \multirow{3}{*}{ V1V5 } & AUC & {$[0.79,0.83]$} & {$[0.82,0.87]$} & {$[0.86,0.90]$} \\
\cline { 2 - 5 } & Sensitividade & {$[0.73,0.79]$} & {$[0.79,0.85]$} & {$[0.84,0.90]$} \\
\cline { 2 - 5 } & Especificidade & {$[0.81,0.87]$} & {$[0.77,0.83]$} & {$[0.81,0.87]$} \\
\hline \multirow{2}{*}{ V1V6 } & AUC & {$[0.78,0.83]$} & {$[0.82,0.86]$} & {$[0.83,0.88]$} \\
\cline { 2 - 5 } & Sensitividade & {$[0.74,0.80]$} & {$[0.79,0.85]$} & {$[0.81,0.86]$} \\
\cline { 2 - 5 } & Especificidade & {$[0.79,0.85]$} & {$[0.76,0.82]$} & {$[0.80,0.86]$} \\
\hline \multirow{2}{*}{ V1V9 } & AUC & {$[0.76,0.81]$} & {$[0.78,0.83]$} & {$[0.81,0.85]$} \\
\cline { 2 - 5 } & Sensitividade & {$[0.70,0.76]$} & {$[0.78,0.85]$} & {$[0.75,0.81]$} \\
\cline { 2 - 5 } & Especificidade & {$[0.80,0.86]$} & {$[0.70,0.77]$} & {$[0.78,0.84]$} \\
\hline
\end{tabular}

TABELA 1 - Análise Multivariada de Quão Bem os Parâmetros Destinguem Entre Pacientes com Câncer de Mama e Controles [Patrício et al. 2018]

\section{TESTES DE CLASSIFICAÇÃO DE PACIENTES UTILIZANDO A REDE NEURAL NEBULOSA}

\subsection{Configuração dos testes}

Nos testes realizados na base de dados foram utilizadas as versões regularizada e não regularizada do modelo de [Souza 2018]. As funções de pertinência são do tipo Gaussianas e Triangulares, os pesos da primeira e da segunda camada foram estimados de forma aleatória no intervalo de $[-3,3]$. Os neurônios utilizados nos testes foram o unineuron e o andneuron. A resposta do modelo é calculada fazendo a conferência da saída da rede com o valor de classificação esperado. O somatório desses valores é dividido pelo total 
de amostras utilizada nas etapas de treinamento e de teste para apurar respectivamente a acurácia. Maiores informações podem ser vistas em [Souza 2018] e [Lemos et al. 2010]. Foram realizadas 30 repetições com as entradas aleatorizadas em cada uma delas para evitar tendências no modelo. O percentual destinado para o treinamento é de $70 \%$ e $30 \%$ para teste. Quando o modelo regularizado foi utilizado, o valor de replicações bootstrap foi de 8 e o consenso de decisão sobre os neurônios foi de 50\%. Esses dois valores foram obtidos após testes prévios utilizando a validação cruzada com valores entre 8, 16, 32 replicações de bootstrap e 50\%, 60\%, 70\% de consenso de decisão. As saídas do modelo foram normalizadas em -1 e 1 para facilitar os devidos cálculos.

\subsection{Testes de Classificação de pacientes}

Para realizar os testes de classificação de padrões para os pacientes de câncer de mama, apresentamos os dois núcleos de testes realizados. Na tabela 2 foi realizado o teste de desempenho da rede neural nebulosa utilizando unineuron com funções de pertinência gaussianas e o andneuron com funções de pertinência triangulares. Os valores de função de pertinência estão no intervalo de [2,3]. Já os valores de acurácia de treinamento e acurácia de teste na tabela 2 são apresentados em valores médios percentuais após as 30 repetições. Os valores indicados em parênteses tratam-se dos desvios padrões para o experimento realizado. Destaca-se também os valores de Especificidade, Sensibilidade e AUC, além da quantidade final de neurônios utilizados após a regularização.

\begin{tabular}{|c|c|c|c|c|c|c|c|}
\hline Base & Modelo & Pertinência & $\begin{array}{l}\text { Qtd Neurônios } \\
\text { Regularizados }\end{array}$ & Especificidade & Sensitividade & AUC & $\begin{array}{l}\text { Acurácia de } \\
\text { Teste }\end{array}$ \\
\hline \multirow{4}{*}{ V1V2 } & \multirow{2}{*}{ UniNet } & 3 & $4,89(2,47)$ & $26,55(8,07)$ & $67,58(10,87)$ & $0,71(0,06)$ & $70,10(6,41)$ \\
\hline & & 3 & $4,86(3,30)$ & $28,42(8,79)$ & $69,85(11,40)$ & $0,71(0,07)$ & $70,20(6,72)$ \\
\hline & \multirow{2}{*}{ AndNet } & 2 & $4(1,70)$ & $41,39(28,89)$ & $76,67(16,30)$ & $0,71(0,07)$ & $69,71(7,79)$ \\
\hline & & 2 & $4,32(1,61)$ & $36,08(22,07)$ & $70,42(15,68)$ & $0,67(0,08)$ & $66,75(7,83)$ \\
\hline \multirow{4}{*}{ V1V3 } & \multirow{2}{*}{ UniNet } & 2 & $8,8(2,35)$ & $24,49(9,32)$ & $68,29(13,65)$ & $0,72(0,08)$ & $71,75(7,67)$ \\
\hline & & 3 & $21,52(10,48)$ & $21,23(7,70)$ & $66,43(8,47)$ & $0,73(0,06)$ & $72,69(5,72)$ \\
\hline & \multirow{2}{*}{ AndNet } & 2 & $7,09(2,91)$ & $26,54(12,03)$ & $66,01(13,23)$ & $0,69(0,07)$ & $68,70(68,70)$ \\
\hline & & 2 & $6,11(2,37)$ & $28,22(10,35)$ & $69,99(15,22)$ & $0,71(0,08)$ & $70,08(7,94)$ \\
\hline \multirow{4}{*}{ V1V4 } & \multirow{2}{*}{ UniNet } & 2 & $24(10,11)$ & $27(9,71)$ & $71,70(10,55)$ & $0,72(0,07)$ & $72,20(7,24)$ \\
\hline & & 3 & $20,43(6,85)$ & $23,62(7,93)$ & $69,62(9,97)$ & $0,73(0,05)$ & $72,55(5,53)$ \\
\hline & \multirow{2}{*}{ AndNet } & 2 & $30,00(15,81)$ & $38,37(22,71)$ & $68,95(18,27)$ & $0,65(0,12)$ & $64,08(11,14)$ \\
\hline & & 3 & $19,03(7,01)$ & $20,67(7,58)$ & $73,32(11,15)$ & $0,77(0,06)$ & $76,65(6,05)$ \\
\hline \multirow{4}{*}{ V1V5 } & \multirow{2}{*}{ UniNet } & 2 & $19,70(7,24)$ & $31,88(9,18)$ & $73,24(9,21)$ & $0,71(0,06)$ & $69,84(5,57)$ \\
\hline & & 3 & $16,27(9,14)$ & $25,38(10,25)$ & $70,46(12,51)$ & $0,69(0,07)$ & $72,09(7,55)$ \\
\hline & \multirow{2}{*}{ AndNet } & 2 & $22,15(6,82)$ & $33,55(15,92)$ & $71,73(13,01)$ & $0,69(0,08)$ & $68,46(8,36)$ \\
\hline & & 3 & $28,03(8,05)$ & $25,59(8,30)$ & $73,36(11,64)$ & $0,68(0,09)$ & $73,10(6,52)$ \\
\hline \multirow{4}{*}{ V1V6 } & \multirow{2}{*}{ UniNet } & 2 & $25,83(7,19)$ & $30,90(7,69)$ & $70,38(12,11)$ & $0,70(0,07)$ & $69,17(7,09)$ \\
\hline & & 3 & $24,27(11,10)$ & $29,21(16,66)$ & $69,68(11,87)$ & $0,68(0,09)$ & $70,11(6,35)$ \\
\hline & \multirow{2}{*}{ AndNet } & 2 & $26,61(7,87)$ & $32,49(6,85)$ & $67,44(12,20)$ & $0,67(0,07)$ & $66,96(6,30)$ \\
\hline & & 3 & $31,61(10,91)$ & $27,94(10,18)$ & $70,39(13,61)$ & $0,66(0,08)$ & $70,20(7,10)$ \\
\hline \multirow{4}{*}{ V1V7 } & \multirow{2}{*}{ UniNet } & 2 & $28,36(8,81)$ & $30,27(12,26)$ & $65,16(9,41)$ & $0,67(0,07)$ & $67,01(7,19)$ \\
\hline & & 3 & $33,69(9,50)$ & $32,34(15,71)$ & $67,70(12,07)$ & $0,65(0,09)$ & $67,80(6,06)$ \\
\hline & \multirow{2}{*}{ AndNet } & 2 & $30,32(8,46)$ & $36,07(20,65)$ & $69,84(14,12)$ & $0,67(0,08)$ & $66,86(7,88)$ \\
\hline & & 3 & $37,08(10,42)$ & $25,20(9,97)$ & $69,96(10,97)$ & $0,64(0,08)$ & $71,65(5,65)$ \\
\hline \multirow{4}{*}{ V1V8 } & \multirow{2}{*}{ UniNet } & 2 & $30,07(8,23)$ & $32,39(15,71)$ & $69,65(12,72)$ & $0,69(0,06)$ & $67,98(5,97)$ \\
\hline & & 3 & $28,43(10,59)$ & $31,86(16,17)$ & $66,90(14,45)$ & $0,69(0,06)$ & $66,83(8,87)$ \\
\hline & \multirow{2}{*}{ AndNet } & 2 & $' 32,19(7,50)$ & $27,93(10,10)$ & $68,12(10,08)$ & $0,70(0,06)$ & $69,56(6,14)$ \\
\hline & & 3 & $46,61(18,07)$ & $34,57(6,40)$ & $70,00(10,83)$ & $0,67(0,07)$ & $67,08(6,35)$ \\
\hline \multirow{4}{*}{ V1V9 } & \multirow{2}{*}{ UniNet } & 2 & $14,65(6,46)$ & $30,82(9,15)$ & $72,07(11,28)$ & $0,71(0,07)$ & $69,56(6,86)$ \\
\hline & & 3 & $22,50(9,66)$ & $24,44(9,20)$ & $69,14(11,55)$ & $0,68(0,07)$ & $71,95(7,03)$ \\
\hline & \multirow{2}{*}{ AndNet } & 2 & $22,58(7,56)$ & $31,03(10,37)$ & $74,89(10,14)$ & $0,72(0,07)$ & $71,32(7,45)$ \\
\hline & & 3 & $23,96(9,57)$ & $27,61(9,60)$ & $72,97(9,60)$ & $0,71(0,09)$ & $72,09(6,97)$ \\
\hline
\end{tabular}

TABELA 2 - Tabela de Desempenho do Teste Rede Neural Nebulosa 
Na tabela apresentada, podemos verificar uma abordagem explícita sobre os conceitos de acurácia e de AUC. O modelo manteve o AUC próximo dos experimentos propostos em [Patrício et al. 2018], divergindo utilizando a média de 30 repetições. A especificidade e a sensibilidade do modelo também auxiliam a entender como a rede neural pode trabalhar com a construção de regras nebulosas evitando falsos positivos negativos. Para o primeiro experimento, com 3 funções de pertinência, podemos atribuir a cada uma delas um valor literal, como "pequeno", "médio" e "grande". Baseado nas funções de pertinência é possível relacionar através de regras SE-ENTÃO os conceitos de Glicose e Resistina, identificando o nível literal de cadas uma delas no paciente, determinando uma correlação que pode servir para a construção de sistemas especialistas.

\section{CONCLUSÕES}

Após os experimentos chegamos à conclusão que mesmo alcançando um valor menor da acurácia em relação ao trabalho de [Lemos et al. 2010], os resultados tem sua relevância para a ciência, pelo motivo da acurácia ser calculada de forma científica e sem tendências. Além disso foi feito um teste mostrando o desvio padrão com 30 repetições que não foi demonstrado no artigo comparado e o uso de um sistema que cria regras nebulosas que ajuda na compreensão dos problemas. Trabalhos futuros podem ser investigados a confirmar a acurácia proposta em [Lemos et al. 2010] nos modelos utilizados. Em testes preliminares nos modelos utilizando a mesma ferramenta e configurações propostas, obteve-se acurácia inferior aos resultados apresentados pela rede neural nebulosa. Outros valores de replicações, consenso e de funções de pertinência podem ser aplicados ao modelo para melhorar os índices avaliados. Outros modelos nebulosos e de redes neurais podem ser submetidos, além de se determinar através de algoritmos de seleção de características, outras combinações mais assertivas para o tratamento de câncer.

\section{Referências}

Bach, F. R. (2008). Bolasso: model consistent lasso estimation through the bootstrap. In Proceedings of the 25th international conference on Machine learning, pages 33-40. ACM.

Bakirtzis, A., Theocharis, J., Kiartzis, S., and Satsios, K. (1995). Short term load forecasting using fuzzy neural networks. IEEE Transactions on Power Systems, 10(3):15181524.

Ballini, R., Soares, S., and Andrade, M. G. (2003). Previsão de vazões médias mensais usando redes neurais nebulosas. Sba: Controle \& Automação Sociedade Brasileira de Automatica, 14(3):680-693.

Bergamasco, R. B. and Angelo, M. (2001). Câncer de mama: Como o diagnóstico é experienciado pela mulher. Revista brasileira de cancerologia, 47(3):277-82.

Calvo, R. (2007). Arquitetura híbrida inteligente para navegação autônoma de robôs. $\mathrm{PhD}$ thesis, Universidade de São Paulo.

de Campos Souza, P. V. and Guimarães, A. J. Utilizando redes neurais nebulosas para melhoria na predição de sistemas especialistas para tratamento de crioterapia.

DeSantis, C., Ma, J., Bryan, L., and Jemal, A. (2014). Breast cancer statistics, 2013. CA: a cancer journal for clinicians, 64(1):52-62. 
Freitas, A. M. S. d., Silva, L. L. d. M. d., Toscani, N. V., and Graudenz, M. S. (2008). Perfil imuno-histoquímico de carcinomas mamários invasores em homens.

Haykin, S. and Network, N. (2004). A comprehensive foundation. Neural networks, 2(2004):41.

Huang, G.-B., Zhu, Q.-Y., and Siew, C.-K. (2006). Extreme learning machine: theory and applications. Neurocomputing, 70(1-3):489-501.

Jang, J.-S. R., Sun, C.-T., and Mizutani, E. (1997). Neuro-fuzzy and soft computing; a computational approach to learning and machine intelligence.

Kasabov, N. et al. (1998). Evolving fuzzy neural networks-algorithms, applications and biological motivation. Methodologies for the conception, design and application of soft computing, World Scientific, 1:271-274.

Lemos, A., Caminhas, W., and Gomide, F. (2010). New uninorm-based neuron model and fuzzy neural networks. In Fuzzy Information Processing Society (NAFIPS), 2010 Annual Meeting of the North American, pages 1-6. IEEE.

Mineo, F. V., Matos, L. d. F. B., da Silva Lima, S., Deluque, A. L., and Ferrari, R. (2013). Assistência de enfermagem no tratamento do câncer de mama. Revista Eletrônica Gestão \& Saúde, 4(2):2238-2260.

Özbay, Y., Ceylan, R., and Karlik, B. (2006). A fuzzy clustering neural network architecture for classification of ecg arrhythmias. Computers in Biology and Medicine, 36(4):376-388.

Patrício, M., Pereira, J., Crisóstomo, J., Matafome, P., Gomes, M., Seiça, R., and Caramelo, F. (2018). Using resistin, glucose, age and bmi to predict the presence of breast cancer. BMC cancer, 18(1):29.

Pedrycz, W. (1991). Neurocomputations in relational systems. IEEE Transactions on Pattern Analysis \& Machine Intelligence, (3):289-297.

Ribeiro-Silva, A. (2012). Core biopsy: uma técnica confiável para o diagnóstico histopatológico do câncer de mama. J Bras Patol Med Lab, 48(1):8-9.

Rossi, L. and dos Santos, M. A. (2003). Repercussões psicológicas do adoecimento e tratamento em mulheres acometidas pelo câncer de mama. Psicologia: ciência e profissão, 23(4):32-41.

Savic, D. A. and Pedrycz, W. (1991). Evaluation of fuzzy linear regression models. Fuzzy sets and systems, 39(1):51-63.

Seabra, Z. T. and Lourenço, J. (2013). Imagiologia no carcinoma da mama. Revista Portuguesa de Cirurgia, (27):59-70.

Silva, A. M., Caminhas, W. M., Lemos, A. P., and Gomide, F. (2013). Evolving neo-fuzzy neural network with adaptive feature selection. In Computational Intelligence and 11th Brazilian Congress on Computational Intelligence (BRICS-CCI \& CBIC), 2013 BRICS Congress on, pages 341-349. IEEE.

Souza, P. V. C. (2018). Regularized fuzzy neural networks for pattern classification problems. International Journal of Applied Engineering Research, 13(5):2985-2991. 